\title{
L'intelligence en essaim. Stratégie d'internationalisation des forums sociaux et régionalisation de la contestation mondiale
}

\section{Raphaël Canet}

\section{(2) OpenEdition}

Journals

Édition électronique

URL : http://journals.openedition.org/conflits/12423

DOI : $10.4000 /$ conflits. 12423

ISSN : $1777-5345$

Éditeur :

CCLS - Centre d'études sur les conflits lilberté et sécurité, L'Harmattan

Édition imprimée

Date de publication : 20 juillet 2008

Pagination : 33-56

ISBN : 978-2-296-05909-2

ISSN : 1157-996X

Référence électronique

Raphaël Canet, «L'intelligence en essaim. Stratégie d'internationalisation des forums sociaux et régionalisation de la contestation mondiale », Cultures \& Conflits [En ligne], 70 | été 2008, mis en ligne le 04 janvier 2010, consulté le 30 mars 2021. URL : http://journals.openedition.org/conflits/12423 ; DOI : https://doi.org/10.4000/conflits. 12423 


\title{
L'intelligence en essaim. Stratégie d'internationalisation des forums sociaux et régionalisation de la contestation mondiale
}

\section{Raphaël CANET}

Raphaël Canet est professeur remplaçant à la faculté des sciences sociales de l'université d'Ottawa (Canada) et cofondateur du collectif de recherche activiste AlterUQAM. Il est membre du secrétariat du Forum social québécois. Ses recherches portent sur la mouvance altermondialiste et la dynamique des forums sociaux, les mobilisations politiques dans les Amériques, la question des identités, les théories de la nation et du nationalisme, la citoyenneté et la gouvernance. Email : canet.raphael@gmail.com

\begin{abstract}
«Ce cycle global de luttes se développe sous la forme d'un réseau réparti. Chaque lutte locale fonctionne sous le modèle d'un point nodal qui communique avec tous les autres sans passer par un centre ou un axe de direction. Chaque lutte reste donc singulière et liée à des conditions locales, tout en étant immergée dans un réseau commun. Ce type d'organisation est ce qui se rapproche le plus d'une expression politique achevée du concept de multitude ${ }^{1}$.
\end{abstract}

\section{Des mondialisations antagoniques}

$\mathrm{F}^{\text {rancis Fukuyama }}{ }^{2}$ lisait dans les décombres du Mur de Berlin la fin du Grand conflit idéologique qui a marqué le $\mathrm{Xx}^{\mathrm{e}}$ siècle. Le triomphe du libéralisme sur le socialisme incarnait la victoire d'une vision du monde sur une autre et allait s'épanouir à mesure que le marché étendrait son empire sur l'ensemble de la planète. Or, force est de constater que la prophétie ne s'est pas réalisée. Alors qu'elle devait apporter la prospérité pour tous, la mondialisa-

1. Negri A., Hardt M., Multitude. Guerre et démocratie à l'âge de l'Empire, Montréal, Boréal, 2004, p. 255.

2. Fukuyama F., La Fin de l'histoire et le dernier homme, Paris, Flammarion, 1992. 
tion néolibérale, essentiellement centrée sur ses dimensions économiques a failli dans sa tâche. Bon nombre de pays - l'Argentine en est le plus bel exemple $^{3}$ - ayant suivi à la lettre les recommandations du Consensus de Washington ${ }^{4}$ en adoptant toutes les politiques macro-économiques censées stimuler la croissance ont été livrés en pâture aux spéculations du capitalisme financiarisé, pourtant entré dans un cycle de crises successives ${ }^{5}$. Depuis le début des années 1990, plus de 80 pays, dont 55 d'Afrique subsaharienne et d'Europe de l'Est, ont vu leur revenu per capita diminuer et, désormais, près de la moitié de la population mondiale vit avec moins de $2 \$$ par jour ${ }^{6}$. Pourtant, l'économie mondiale n'a jamais été aussi prospère. Le Produit brut mondial a plus que doublé ces trente dernières années pour atteindre, au tournant des années 2000, plus de 30000 milliards de dollars 7 .

Ce n'est pas la fin de l'Histoire qu'annonçait le terme de la Guerre froide, mais bien son recommencement, alors que se redéfinissaient les espaces, les acteurs, les modes de régulation et les formes de mobilisation sur la scène internationale ${ }^{8}$. A la mondialisation ${ }^{9} \mathrm{du}$ capital financier a répondu la mondialisation de la résistance des peuples qui a connu diverses formes d'actualisation. D’une attitude défensive et d'opposition affichée dès la fin des années 1990, lors des contre-sommets et autres manifestations tenues en marges des grands rassemblements officiels des organisations internationales à caractère économique (Organisation mondiale du commerce, Fonds monétaire international, Banque mondiale, G8), le mouvement pour une justice mondiale 10 a progressivement évolué vers une position plus offensive de proposition, notamment

3. Chesnais F., Divès J.-P., ; Que se vayan todos! Le peuple d'Argentine se soulève, Paris, Nautilus, 2002. Voir aussi le documentaire de Fernando E. Solanas, Memoria del saqueo, 2003.

4. Williamson J., "What Washington means by policy reform", in Williamson J. (ed.), Latin American Adjustment: How Much Has Happened, Washington, Institute for International Economics, 1990, pp. 7-38.

5. Boyer R., Dehove M., Plihon D., Les Crises financières, Paris, La Documentation française, Les rapports du Conseil d'analyse économique, ${ }^{\circ} 50,2004$.

6. Association québécoise des organismes de coopération internationale, Actes des Etats généraux de la coopération et de la solidarité internationales, Québec, Montréal, novembre 2006. Disponible en ligne : www.aqoci.qc.ca

7. Organisation de coopération et de développement économiques, OCDE, www.oecd.org

8. La littérature est très vaste sur ces thèmes, nous nous référons ici, et sur différents registres, à : Badie B., Smouts M.-C., Le Retournement du monde, Paris, Presses de la FNSP, 1999; Rosenau J., Along the Domestic-Foreign Frontier: Exploring Governance in a Turbulent World, Cambridge, Cambridge University Press, 1997 ; Laroche J., Politique internationale, Paris, LGDJ, 2000 ; Badie B., La Fin des territoires, Paris, Fayard, 1995 ; Appadurai A., Après le colonialisme. Les conséquences culturelles de la globalisation, Paris, Payot, 2001 ; O’Brien R., Goetz A.-M., Scholte J.A., Contesting Global Governance: Multilateral Economic Institutions and Global Social Movements, Cambridge, Cambridge University Press, 2000 ; Chevallier J., L’Etat postmoderne, Paris, LGDJ, coll. « Droit et société », n³5, 2003.

9. Nous comprenons le concept de mondialisation, à la manière de l'économiste CharlesAlbert Michalet, comme la troisième configuration de la mondialisation qui a émergé au milieu des années 1980 et qui consacre le discours néolibéral se fondant sur la "proclamation véhémente que les marchés doivent devenir le principe exclusif de la régulation ". Michalet C.A., Qu'est-ce que la mondialisation ?, Paris, La Découverte/Poche, 2004, p. 95.

10. George S., Un autre monde est possible si..., Paris, Fayard, 2004. 
dans le cadre du Forum social mondial (FSM) dont la première édition s'est tenue à Porto Alegre (Brésil), du 25 au 30 janvier 200111.

Qu'elle soit définie en termes de contre-pouvoir ${ }^{12}$, de mondialisation contre-hégémonique ${ }^{13}$, de mouvement anti-systémique ${ }^{14}$ ou encore de berceau d'une hypothétique Cinquième Internationale ${ }^{15}$, la mouvance altermondialiste s'est imposée dans le champ de la représentation en brisant le dogme de l'unicité de la pensée, du modèle de développement, de la rationalité instrumentale. Derrière le slogan «Un autre monde est possible », se dessine une nouvelle utopie rêvant d'une :

«mondialisation solidaire qui respecte les droits universels de l'Homme, ceux de tous les citoyens et citoyennes de toutes les nations, et l'environnement, soutenue par des systèmes et institutions internationaux démocratiques au service de la justice sociale, de l'égalité et de la souveraineté des peuples ${ }^{16}$. »

Cette nouvelle utopie est porteuse d'espoir pour les mouvements qui s'en réclament. Cependant, considérant que la mondialisation est le produit de plus de trente ans d'hégémonie idéologique et politique du néolibéralisme dont le Forum économique mondial de Davos ${ }^{17}$ s'est fait le relais ${ }^{18}$, le défi que doit relever la mouvance altermondialiste est immense. Véritable travail de Sisyphe, cette élaboration d'alternatives se situe par ailleurs dans un contexte idéologique et géopolitique caractérisé par le naufrage des grandes utopies socialistes, dont la chute du Mur de Berlin constitue le symbole le plus éclatant.

11. Whitaker C., Changer le monde. Nouveau mode d'emploi, Paris, Les éditions de l'Atelier, 2006 ; Agrikoliansky E., Fillieule O., Mayer N. (dirs.), L'Altermondialisme en France : la longue histoire d'une nouvelle cause, Paris, Flammarion, 2005.

12. Beck U., Pouvoir et contre-pouvoir à l'ère de la mondialisation, Paris, Flammarion, Aubier, 2003.

13 . de Sousa Santos B., "Nuestra América. Reinventing a subaltern paradigm of recognition and redistribution", Theory, Culture E Society, vol.18, n²-3, 2001, pp. 185-218.

14. Wallerstein I., "Que signifie aujourd'hui un mouvement antisystémique ?», in Wieviorka M. (dir.), Un autre monde... Contestations, dérives et surprises dans l'antimondialisation, Paris, Balland, 2003, pp. 93-105.

15. Amin S., Pour la Cinquième Internationale, Paris, Le Temps des Cerises, 2006.

16. Article 4 de la Charte des principes du Forum social mondial, adoptée par le Conseil international du FSM le 10 juin 2001. Texte en ligne sur le site du FSM :

www.forumsocialmundial.org.br

17 . Digne héritier de la société du Mont-Pèlerin (1947) et du colloque Lippman (1938), le Forum économique mondial se tient chaque année depuis 1971. Lemaître F., "Forum économique mondial : des élites en quête de sens ", Le Monde, 22 janvier 2008.

18. Comme le souligne Dorval Brunelle, "La notion de relais [appliquée au Forum de Davos] sert ici à mettre en relief l'émergence d'un lieu nouveau où se retrouvent et se côtoient hommes politiques et gens d'affaires à l'occasion de rencontres au cours desquelles les seconds avancent des propositions que les premiers sont invités à suivre afin de favoriser l'essor d'une économie globale ». Brunelle D., "Le Forum social mondial : origine et participants ", Chronique des Amériques, $\mathrm{n}^{\circ} 3$, janvier 2006, p. 2. En ligne : www.ameriques.uqam.ca 
La mouvance altermondialiste a, dès lors, tenté de tenir compte de cette leçon de l'histoire en faisant le pari de la force de changement du multiple. Si l'alternative est toujours possible, elle ne doit ni se penser au singulier, ni découler d'une vision qui émanerait d'une avant-garde éclairée et qui se diffuserait vers les masses opprimées alors soudainement éveillées à leur conscience de classe. Au contraire, ces projets alternatifs doivent plutôt émaner de la base des mouvements sociaux ${ }^{19}$. Plutôt que la construction d'un système de pensée surplombant et cohérent, c'est la manifestation de la diversité des points de vue et des initiatives que favorise la mouvance altermondialiste. Cet a priori envers la vertu du multiple, de la diversité des luttes et des pratiques pose à la mouvance altermondialiste le défi de la cohérence. Comment mettre en acte cette utopie ? Afin de pouvoir asseoir la dynamique du changement social sur la diversité des pratiques plutôt que sur une élaboration théorique, il fallait faire preuve d'innovation. Le Forum social mondial, conçu comme un lieu de réflexion et d'échange sur l'altermondialisme, un espace de convergence de la diversité des pratiques alternatives, est alors apparu comme le creuset de cette mondialisation alternative ${ }^{20}$. Le Forum social mondial a tout d'abord été élaboré en s'opposant au modèle du Forum économique mondial. Par le nom, la date et le lieu, il s'agissait de construire l'anti-modèle de Davos ${ }^{21}$. Puis, afin de favoriser l'essor de la mouvance altermondialiste en s'appuyant sur cette innovation politique, le FSM a développé sa propre dynamique. Tout d'abord, il y a eu l'adoption de la Charte des principes 22 (avril-juin 2001) dans laquelle se trouve consigné un socle de valeurs communes permettant de rassembler, dans une vision partagée du monde et de son devenir souhaité, une large diversité de groupes, de mouvements, d'individus. Ensuite, en 2002 est déployée une stratégie «de mondialisation et d'enracinement » des forums sociaux ${ }^{23}$. Celle-ci a principalement consisté à favoriser le processus d'expansion du mouvement en y intégrant la diversité des revendications émergentes tout autour du monde, ce qui signifiait à la fois délocaliser le Forum social mondial 24 et stimuler l'organisation de forums régionaux ${ }^{25}$.

19. Portes A., "Globalization from below: the rise of transnational communities", Working Paper of Transnational Communities Programme, Princeton University, septembre 1997. En ligne : www. www.transcomm.ox.ac.uk

20. Pleyers G., «Les Forums sociaux comme modèle idéal de convergence », Revue internationale des sciences sociales, ${ }^{\circ} 182,2004$, pp.569-579.

21. Sur les origines du FSM, Cassen B., Tout a commencé à Porto Alegre... Mille forums sociaux!, Paris, Mille et une nuits, 2003 ; Whitaker C., Changer le monde. Nouveau mode d'emploi, Paris, Editions ouvrières, 2006.

22. La Charte des principes du Forum social mondial est le document de base du FSM. Tous ceux qui veulent organiser des forums sociaux de façon intégrée à la dynamique du FSM, à quelque échelle que ce soit, doivent s'y référer. Elle est disponible en ligne sur le site du FSM : www.forumsocialmundial.org.br

23. Corrêa Leite J., Fórum Social Mundial. A história de uma invenção política, São Paulo, Editora Fundaçao Perseu Abramo, 2003, voir notamment le chapitre 5 : «A mundialização e o futuro do Fórum Social Mundial », pp. 109-126.

24. Après trois éditions au Brésil (2001, 2002 et 2003), le FSM s'est délocalisé en Inde (2004), pour revenir au Brésil (2005), adopter une formule polycentrique en 2006 (Mali-Vénézuela-Pakistan), opter pour l'Afrique (Kenya, 2007), et finalement adopter une formule totalement décentralisée en 2008 (une journée mondiale d'action). Le FSM reviendra au Brésil en 2009 (Bélem).

25 . Depuis 2002, se sont déroulés des Forums sociaux pan-amazoniens, européens, africains, asia- 
Ce texte vise à explorer les apparents paradoxes et possibles limites de ce processus d'expansion du Forum social mondial en questionnant la dimension globale de la mouvance altermondialiste. En tentant de dresser un parallèle avec la logique de la mondialisation économique qui, par-delà son discours globaliste, a conduit effectivement à la construction de blocs économiques régionaux, nous souhaitons explorer l'hypothèse selon laquelle la mobilisation altermondialiste, au-delà du discours rassembleur de la contestation du néolibéralisme et de l'impérialisme, se décline elle aussi en des particularismes régionaux. En d'autres termes, sous couvert d'un discours faisant la promotion de la transnationalisation de l'action collective dans le cadre de l'altermondialisme ${ }^{26}$, nous assisterions à une régionalisation à la fois des thématiques et des formes d'action.

Nous présenterons les fondements axiologiques de la mouvance altermondialiste ainsi que les différentes logiques qui alimentent le processus d'expansion des forums sociaux. Puis, à partir d'une analyse qualitative comparée des programmes et déclarations de cinq forums sociaux ${ }^{27}$, croisée avec le fruit de nos observations sur le terrain, nous illustrerons cette tendance à la régionalisation de la contestation mondiale en dégageant les lignes de force des différents particularismes. Cela nous permettra de revenir sur la question de l'efficacité de cette stratégie et surtout sur la compréhension de la démarche altermondialiste. La faible couverture médiatique des forums sociaux, leur manque de visibilité et surtout de lisibilité, sont souvent interprétés comme des indices du déclin, de l'essoufflement, voire de l'oubli de la mouvance altermondialiste. Adopter ce point de vue nous semble réducteur, car il omet le fait que les mondialisations antagoniques sont avant tout le produit de luttes symboliques et que l'arène médiatique n'est ni neutre ${ }^{28}$, ni l'unique espace d'expression de la réalité sociale.

\section{La mondialisation des forums sociaux}

\section{Les fondements axiologiques de l'utopie altermondialiste}

L'utopie altermondialiste repose sur un socle de valeurs communes qui assurent la cohésion de la mouvance qui investit les forums sociaux ${ }^{29}$. Quatre grandes orientations axiologiques peuvent être dégagées de la Charte des principes du FSM.

tiques, des Amériques, de la Méditerranée, des Caraibes... Ainsi que des centaines de forums sociaux nationaux et locaux.

26. Della Porta D., Tarrow S.(eds.), Transnational protest and global activism, Lanham, Rowman \& Littlefield, 2005 ; Bandy J., Smith J., Coalitions across Borders. Transnational protest and the neoliberal order, Lanham, Rowman \& Littlefield, 2005.

27. Il s'agit du $2^{\mathrm{e}}$ Forum social des Amériques (Quito, 2004), du $3^{\mathrm{e}}$ Forum social européen (Londres, 2004), des 4e et $5^{\mathrm{e}}$ Forums sociaux mondiaux (Mumbai, 2004 et Porto Alegre, 2005) et du $1^{\mathrm{er}}$ Forum social méditerranéen (Barcelone, 2005).

28. Champagne P., Faire l'opinion. Le nouveau jeu politique, Paris, Editions de Minuit, 1990.

29. Ces fondements axiologiques ont déjà été exposés ailleurs : Canet R., "L'éclosion d'une culture politique participative. L'expérience du Forum social québécois », Nouvelles pratiques sociales, vol.21, $\mathrm{n}^{\circ} 1$, automne 2008 . 
Tout d'abord, on a le rejet du néolibéralisme compris dans le discours des acteurs comme le fondement idéologique de la mondialisation capitaliste qui sert les intérêts mercantiles des entreprises multinationales. C'est à la fois l'architecture économique et financière mondiale, le mode de développement capitaliste et les rapports sociaux qu'il suppose qui sont ciblés et dénoncés, au profit d' "une mondialisation solidaire qui respecte les droits universels de l'Homme, ceux de tous les citoyens et citoyennes de toutes les nations, et l'environnement 30 ", et qui met au centre du projet de société à construire les valeurs de justice sociale, d'égalité et de souveraineté des peuples.

On trouve ensuite la lutte contre l'impérialisme. Cette deuxième dimension axiologique a quelque peu évolué depuis 2001, notamment du fait de l'invasion américaine en Irak et des importantes marches contre la guerre qui, depuis le printemps 2003, se répètent chaque année dans de nombreux pays ${ }^{31}$. Initialement articulée autour d'une définition assez large du concept, à savoir «l'usage de la violence comme moyen de contrôle social par l'Etat ", débouchant sur des «formes de domination comme l'assujettissement d'un être humain par un autre 32 ", l'impérialisme, comme avatar du capitalisme, fut remis à l'avant-scène avec la guerre en Irak, tout en éclairant d'un jour nouveau le conflit israélo-palestinien ${ }^{33}$.

L'éloge de la diversité constitue une autre valeur fondamentale de la mouvance altermondialiste. Cette posture vise à s'extirper de la tendance unitaire et homogénéisante de la pensée unique. Elle permet de concevoir la diversité, non comme une source de fragmentation et de division, véhiculée par une vision du monde fondée sur la concurrence et la rivalité, mais bien comme une profonde richesse permettant un partage d'expérience et une interfécondation des savoirs menant à une véritable solidarité. Il s'agit donc de rompre avec l'idée d'un modèle unique de société qui s'impose à tous et de reconnaître la diversité non hiérarchisée des expériences et des modes d'organisation sociale. C'est pour cela que le FSM s'affiche comme «un espace pluriel et diversifié, non confessionnel, non gouvernemental et non partisan, qui articule de façon décentralisée, en réseau, des instances et mouvements engagés dans des actions concrètes, au niveau local ou international, visant à bâtir un autre monde ${ }^{34}$ ».

Enfin, une conception horizontale des relations de pouvoir qui, d'une part, rejette la théorie de son monopole associée à la notion de souveraineté absolue qui a conduit à l'institutionnalisation de l'Etat moderne et, d'autre

30. Charte des principes du FSM, article 4.

31 . Les premières marches contre la guerre, organisées en février 2003, ont rassemblé près de 10 millions de personnes dans plus de 60 pays. Les manifestations se répètent chaque année, mais avec des mobilisations moins importantes.

32. Charte des principes du FSM, article 10.

33. Ce fut d'ailleurs l'un des thèmes majeurs du Forum social thématique palestinien qui s'est tenu à Ramallah, en Cisjordanie, du 27 au 30 décembre 2002.

34. Charte des principes du FSM, article 8. 
part, refuse le mode d'organisation pyramidal fondé sur un principe hiérarchique conduisant à la distinction entre élite et masse. Finalement, cette conception débouche sur une critique du principe de délégation du pouvoir qui fonde la légitimité des démocraties représentatives et qui conduit à une forme passive de citoyenneté. A l'inverse, cette théorie de l'horizontalité s'articule autour de la conception d'un pouvoir qui s'est diffusé dans l'ensemble sociétal. Elle suppose un mode d'organisation réticulaire répondant au principe d'agrégation des initiatives et des actions émanant d'une multitude complexe d'acteurs sociaux et elle débouche sur une ouverture aux différentes modalités de la démocratie participative ${ }^{35}$ qui mettent en avant les principes d'inclusion et de citoyenneté active.

\section{La stratégie d'internationalisation des forums sociaux}

Une fois ces valeurs centrales de cohésion définies et affirmées, la mouvance altermondialiste se devait de les propager, de les partager et de les pratiquer de manière à concrétiser sa prétention planétaire et à construire les alternatives, d'où la stratégie de diffusion des forums sociaux, afin de rallier un nombre sans cesse croissant de participants pour que puissent s'y manifester les formes les plus diversifiées de luttes sociales qui parcourent la planète et s'y forger de nouvelles alliances transnationales entre mouvements sociaux. Cette stratégie d'internationalisation du FSM peut être déclinée selon quatre logiques : délocalisation, multiplication, déclinaison et diversification ${ }^{36}$.

\section{La logique de délocalisation du Forum social mondial}

Fruit d'une initiative franco-brésilienne ${ }^{37}$, les trois premières éditions du FSM se sont tenues à Porto Alegre au Brésil. Il s'agissait de poser un acte symbolique suffisamment fort pour incarner l'émergence d'une alternative au Forum économique mondial qui se tient depuis 1971 à Davos (Suisse). Ainsi, Porto Alegre, cette ville du Sud qui expérimente de nouvelles formes de participation politique, est apparue comme le berceau idéal des forums sociaux ${ }^{38}$. Mais, pour correspondre à son ambition globaliste, le FSM ne pouvait demeurer cantonné à son foyer brésilien. Ainsi, en 2004, le Forum s'est délocalisé à

35. Sur ce concept de démocratie participative, voir notamment Gret M., «Brésil : une autre manière de gouverner au local ? ", in Monclaire S., Deluchey J.-F. (dirs.), Gouverner l'intégration : politique nationale et internationale du Brésil de Lula, Paris, éditions Pepper, 2006, chap. 2 ; Bacqué M.-H., Rey H., Sintomer Y., Gestion de proximité et démocratie participative, une perspective comparative, Paris, La Découverte, 2005 ; Barthe Y., Callon M., Lascoumes P., Agir dans un monde incertain, introduction à la démocratie technique, Paris, Seuil, 2001 ; de Sousa Santos B. (dir.), Democratizar a democracia. Os Caminhos da democracia participativa, Rio de Janeiro, Civilização Brasileira, 2002.

36. Ces logiques ont déjà été décrites plus longuement dans Canet R., Perrault S., « Les forums sociaux : vers une culture politique de l'implication citoyenne », in Canet R., Duchastel J. (dirs.), Crise de l'Etat, revanche des sociétés, Montréal, Athéna éditions, 2006, pp. 254-268.

37. Cassen B., op. cit.

38. Gret M., Sintomer Y., Porto Alegre. L'espoir d'une autre démocratie, Paris, La Découverte, 2002. 
Mumbai, en Inde. Ce changement de lieu visait à rallier les groupes opprimés du continent asiatique, notamment le mouvement des Dalits ${ }^{39}$.

Puis, le FSM est revenu à son bercail du Rio Grande do Sul en 2005, pour connaître son plus grand succès de participation, plus de 150000 personnes. Il est ensuite, et pour la première fois, polycentrique en 2006. Cette année-là en effet, trois forums sociaux mondiaux ont eu lieu consécutivement dans trois régions du monde : Venezuela (Caracas), Mali (Bamako) et Pakistan (Karachi). En 2007, le FSM s'est reconcentré en un seul lieu, à Nairobi (Kenya). En 2008, c'est une formule totalement décentralisée qui a été adoptée. Plutôt qu'un FSM, un appel à une journée d'action mondiale pour le 26 janvier a été lancé. Dans l'état actuel des bilans, il semblerait que cet appel ait débouché sur plus de 800 activités dans 80 pays et qu'il ait mobilisé plus de 2500 organisations ${ }^{40}$. Le prochain FSM aura lieu en janvier 2009 à Bélem (Brésil).

Ce processus de délocalisation du FSM, associé aux multiples formules innovantes (polycentrique, décentralisée) qui ont été expérimentées, vise à permettre une plus grande intégration des peuples du monde qui demeurent encore trop marginalisés au sein de la mouvance altermondialiste (notamment en Asie, en Europe de l'Est et en Russie).

\section{La logique de multiplication des forums sociaux}

Parallèlement à cette logique de délocalisation du Forum social mondial, les organisateurs ont initié une logique de multiplication des forums sociaux. En effet, divers types de forums sont organisés à travers le monde. Evidemment, le plus important et le plus médiatisé reste le FSM. Mais, chaque année, plusieurs forums s'organisent aux échelles régionale, nationale et locale, sans compter les forums thématiques.

Ces divers types de forums entendent permettre tout d'abord aux activistes les plus engagés de multiplier les tribunes, de se mobiliser à plusieurs reprises au cours d'une même année et, ainsi, d'élargir leurs réseaux et de diversifier leurs stratégies d'action. Ils souhaitent également donner à ceux qui n'ont pas la chance de pouvoir se déplacer d'un pays à un autre la possibilité de prendre part aux débats dans un lieu plus proche. Ces rencontres de plus petite taille offrent

39. Les Dalits sont les populations qui constituent dans le système des castes indien les «Intouchables », ou « hors-castes ». Ils forment plus de $17 \%$ de la population (soit 170 millions de personnes) et ont choisi eux-mêmes de porter ce nom, qui signifie « opprimé » en Marathi. Couche la plus marginalisée de la société indienne, ils ont profité de la venue du FSM en Inde pour développer leur force revendicatrice en forgeant des réseaux transnationaux de solidarité avec leur cause. Smith P. "The Transnational Politics of the Dalit Movement”, Paper presented at the annual meeting of the Midwest Political Science Association, Palmer House Hilton, Chicago, Illinois, 20 avril 2006.

40. Pour plus d'informations sur cette journée mondiale d'action : http://wsf2008.net 
enfin la possibilité aux participants d'exprimer leurs revendications particularistes en discutant de thématiques locales et régionales, leur donnant l'opportunité de trouver des solutions plus adaptées aux problèmes auxquels ils font face, à des échelles d'action plus réduites. Dans cette perspective, la multiplication des forums, à différentes échelles d'action, permet aux citoyens actifs de se mobiliser sur plusieurs fronts et de débattre d'enjeux à la fois locaux, régionaux et mondiaux. Ainsi, le travail d'élargissement de la participation et de la mobilisation passe plus par les forums locaux, régionaux et thématiques que par le FSM. Cette initiative de multiplication des forums repose sur la stratégie du mouvement selon l'axiome suivant : penser global, agir local.

\section{La logique de déclinaison des forums}

La troisième logique mise en œuvre est celle de la déclinaison des forums, c'est-à-dire la tenue simultanée de plusieurs forums sectoriels en marge du FSM. C'est ainsi que pour la première fois à Porto Alegre, du 26 au 31 janvier 2005, sept forums sectoriels se sont tenus en même temps que le FSM ${ }^{41}$, tous à Porto Alegre du 26 au 31 janvier.

Cette stratégie de déclinaison a pour objectif de favoriser les rencontres et discussions entre divers groupes autour d'activités précises, mais surtout d'approfondir une réflexion technique sur des enjeux particuliers. Ainsi, ce type d'événement vise à faciliter la convergence entre des acteurs ayant des intérêts communs et à engendrer une spécialisation des forums. En outre, les forums sectoriels permettent d'attirer des gens qui, autrement, considéreraient l'événement mondial comme une perte de temps. La tenue simultanée des forums encourage enfin la rencontre avec les participants du FSM, élargissant ainsi le spectre des thèmes abordés. Malheureusement, étant donné le grand nombre d'ateliers et le programme chargé des journées de ces forums, la multiplication des événements rend finalement difficile la gestion de la diversité.

\section{La logique de diversification des thématiques}

Comme la précédente, la logique de diversification des thématiques a donné lieu à une innovation majeure au cours du Forum social mondial de 2005. En effet, cette diversification s'est matérialisée dans des espaces thématiques distincts, conceptuellement mais aussi géographiquement. Onze espaces thématiques ont été répartis sur l'ensemble du « Territoire social mon-

41. Il s'agit du $1^{\text {er }}$ Forum social des migrations, du $1^{\text {er }}$ Forum social mondial de la santé, du $4^{\mathrm{e}}$ Forum mondial des magistrats, du $4^{\mathrm{e}}$ Forum des autorités locales pour l'inclusion sociale, du $1^{\text {er }}$ Forum mondial de l'information et de la communication, du $5^{\mathrm{e}}$ Forum parlementaire mondial et enfin, du $4^{\mathrm{e}}$ Forum syndical mondial. On remarque que ces forums parallèles se classent en deux catégories : la première est celle des activités (migrations, santé et information et communication), la seconde est celle des acteurs (magistrats, autorités locales, parlementaires et syndicats). 
dial », étalé sur 5 kilomètres le long du fleuve qui borde la ville. Ces espaces séparés ont constitué autant d'îlots de réflexion spécifiques sur des thèmes particuliers. Nous retrouvons ici la logique de déclinaison, mais qui se développe cette fois à l'intérieur du FSM, et non en parallèle.

Ces quatre logiques brièvement présentées ici démontrent la volonté des artisans du FSM de promouvoir la plus large inclusion possible au sein de la mouvance altermondialiste. Ce processus d'inclusion s'est effectué selon deux dimensions. D'abord, sur le plan géographique, la formule des forums sociaux a évolué selon une dynamique d'expansion (logique de délocalisation du FSM), mais aussi d'approfondissement vers différentes échelles d'action (logique de multiplication des forums sociaux). Ensuite, sur le plan thématique, s'est opéré, à l'intérieur même du FSM, un processus de diversification, renforçant ainsi sa dynamique d'expansion; puis, en marge du FSM, la logique de déclinaison des forums sectoriels a complété son approfondissement en traitant plus spécifiquement de thématiques particulières. Ce double processus de déplacement géographique et thématique a ainsi permis de circonscrire un espace inclusif de débat citoyen global, une sorte d'espace discursif et programmatique mondial alternatif dont il convient désormais d'analyser la cohérence.

\section{La régionalisation de la contestation mondiale}

Ce processus d'expansion des forums sociaux a permis l'éclosion d'une multiplicité de lieux de débat, de rencontre, d'échange, de mise en réseau, de pratiques alternatives. Cependant, nous sommes en droit de nous interroger sur les incidences de ce processus sur la cohérence d'ensemble de l'action globale. En effet, l'éloge de la diversité et la volonté d'une inclusion sans cesse plus poussée ne vont-elles pas conduire à une trop grande dispersion rendant impossible toute tentative de tirer un plan d'action clair et cohérent de cette masse disparate de propositions parfois contradictoires ? En somme, le processus d'élargissement et d'approfondissement de cet espace mondial de dialogue, d'échange et d'élaboration d'actions concertées ne conduit-il pas à une fragmentation de la mouvance altermondialiste et à une dissipation des énergies rendant peu probable une véritable inflexion du cours de la mondialisation?

\section{Analyse des thématiques}

Afin de mettre à l'épreuve notre hypothèse de la régionalisation de la contestation mondiale, nous nous sommes livrés à une analyse des thématiques abordées au cours de cinq forums sociaux qui se sont déroulés entre 2004 et 2005, à savoir les Forums sociaux mondiaux de Mumbai (FSM, 16-21 janvier 2004) et de Porto Alegre (FSM, 26-31 janvier 2005), le Forum social des Amériques de Quito, Equateur (FSA, 26-30 juillet 2004), le Forum social européen de Londres (FSE, 14-17 octobre 2004) et le Forum social de la Méditerranée de Barcelone (FSMed, 16-19 juin 2005). Nous nous sommes 
concentrés sur cette fenêtre historique relativement étroite, car celle-ci permet l'analyse comparative relativement synchronique (ces cinq forums se sont déroulés en l'espace de dix-huit mois sur trois continents différents) d'événements dotés d'un fort potentiel innovant (Porto Alegre a été le FSM le plus fréquenté de la jeune histoire de la mouvance, Mumbai correspondait au premier FSM situé hors du Brésil, et quant aux forums de Barcelone et de Quito, ils ont constitué des premières au niveau régional).

Avant même de procéder à une analyse qualitative plus poussées des programmes, un premier constat s'impose : le nombre d'axes thématiques proposés varie sensiblement d'un forum à un autre.

\begin{tabular}{|c|c|c|c|c|}
\hline FSM 2004 & FSA 2004 & FSE 2004 & FSM 2005 & FSMed 2005 \\
\hline 4 axes & 5 axes & 6 axes & 11 axes & 7 axes \\
\hline
\end{tabular}

Cette diversité tient au fait que chacun des forums met en place son propre comité d'organisation, lequel définit, le plus souvent au sein de son comité sur les contenus et les thèmes et selon un processus plus ou moins large de consultation, les grands axes thématiques de l'événement. Il revient ensuite à chaque groupe participant d'inscrire son activité sous la thématique générale définie par l'organisation : c'est ce que l'on nomme le processus d'auto-programmation. Remarquons cependant que le nombre d'axes thématiques ne préfigure pas de la pauvreté ou de la richesse des débats qui vont être menés au sein du forum, mais rend plutôt compte de la définition plus ou moins large des problématiques mises à l'ordre du jour par le comité local d'organisation.

Chacun des axes thématiques des forums étudiés se décline sous une forme plus ou moins développée. Afin de faciliter leur comparaison, nous les avons regroupés par catégories. L'étude des trente-trois axes thématiques énoncés au cours de nos cinq forums nous a permis de définir huit catégories : néolibéralisme économique, impérialisme, démocratie et droits, culture et communication, groupes particuliers, migrations, environnement et développement, religion. Nous disposons de cette façon d'une base comparative afin d'évaluer les convergences et divergences thématiques de chacun des forums et ainsi de distinguer les thèmes globaux des thèmes régionaux.

\section{Les thèmes globaux}

Les thèmes globaux sont ceux dont les catégories figurent dans tous les forums observés. Nous pouvons ainsi identifier cinq thèmes globaux. 


\begin{tabular}{|c|c|}
\hline FSM 2004 & $\begin{array}{l}1 \text { - Militarisme, guerre et agression } \\
2 \text { - Médias, information et savoirs } \\
3 \text { - Démocratie, sécurité écologique et économique } \\
4 \text { - Exclusion, dignité et droits } \\
\text { Thèmes transversaux : genre et diversité }\end{array}$ \\
\hline FSA 2004 & $\begin{array}{l}1 \text { - L’ordre économique } \\
2 \text { - Le caractère violent du projet néolibéral } \\
3 \text { - Pouvoir, démocratie et Etat } \\
4 \text { - Cultures et communication } \\
5 \text { - Peuples indigènes et afro-américains }\end{array}$ \\
\hline FSE 2004 & $\begin{array}{l}1 \text { - Paix et guerre } \\
2 \text { - Démocratie et droits fondamentaux } \\
3 \text { - Justice sociale et solidarité : contre la privatisation et pour les } \\
\text { droits sociaux des travailleurs et des femmes } \\
4 \text { - Mondialisation des entreprises et justice globale } \\
5 \text { - Contre le racisme, la discrimination et pour des droits équita- } \\
\text { bles : pour l'égalité et la diversité } \\
6 \text { - Crise environnementale, contre le néolibéralisme et pour une } \\
\text { société durable }\end{array}$ \\
\hline FSM 2005 & $\begin{array}{l}1 \text { - Pensée autonome, réappropriation et socialisation des savoirs } \\
\text { et des technologies } \\
2 \text { - Défendre la diversité, la pluralité et les identités } \\
3 \text { - Art et création : construire des cultures de résistance popu- } \\
\text { laire } \\
4 \text { - Communication : pratiques anti-hégémoniques, droits et } \\
\text { alternatives } \\
5 \text { - Assurer et défendre des biens communs de la Terre et du } \\
\text { peuple comme alternative à la marchandisation et à la domina- } \\
\text { tion des entreprises transnationales } \\
6 \text { - Luttes sociales et alternatives démocratiques contre la domi- } \\
\text { nation néolibérale } \\
7 \text { - Paix, démilitarisation et lutte contre la guerre, le libre- } \\
\text { échange et la dette } \\
8 \text { - Vers la construction d'un ordre démocratique international et } \\
\text { l'intégration des peuples } \\
9 \text { - Economies souveraines pour et par les peuples contre le capi- } \\
\text { talisme néolibéral } \\
10 \text { - Droits humains et dignité pour un monde juste et égalitaire } \\
11 \text { - Ethique, cosmovision et spiritualité }\end{array}$ \\
\hline FSMed 2005 & $\begin{array}{l}1 \text { - Démocratie, citoyenneté et droits humains des hommes et } \\
\text { des femmes } \\
2 \text { - Conflits, occupations militaires, militarisme et paix } \\
3 \text { - Droits économiques, sociaux et culturels, modèles de } \\
\text { développement, travail et conflits } \\
4 \text { - Migrations } \\
5 \text { - Diversité culturelle et dialogues transculturels } \\
6 \text { - Modèle de développement et environnement } \\
7 \text { - Femmes et Méditerranée }\end{array}$ \\
\hline
\end{tabular}


La catégorie de l'impérialisme rassemble l'ensemble des thématiques liées à la guerre, aux conflits, au militarisme. Cette thématique est omniprésente sur la planète et figure au centre du discours altermondialiste comme le versant violent et brutal du néolibéralisme, notamment depuis le déclenchement de la guerre en Irak.

La catégorie du néolibéralisme économique regroupe tous les thèmes relevant de l'ordre économique, du libre-échange, de la question de la dette, du capitalisme, du monde du travail... Là encore, il n'y a pas de réelle surprise, puisque c'est l'argument majeur de la critique de la mondialisation néolibérale.

La catégorie démocratie et droits rassemble toutes les thématiques relevant du politique, de l'Etat, de la démocratie et surtout de la question des droits, qui tendent à se décliner sous une multiplicité de formes, des droits fondamentaux aux plus particularistes.

Tableau des catégories :

Viennent enfin deux catégories également globales, puisqu'elles se trouvent abordées dans la totalité des forums. Cependant une nuance doit être formulée puisque ces catégories ne sont envisagées que comme des éléments secondaires pour deux forums.

\begin{tabular}{|c|c|c|c|c|c|}
\hline Catégories & FSM 2004 & FSA 2004 & FSE 2004 & FSM 2005 & FSMed 2005 \\
\hline Impérialisme & $\mathrm{X}$ & $\mathrm{X}$ & $\mathrm{X}$ & $\mathrm{X}$ & $\mathrm{X}$ \\
\hline $\begin{array}{l}\text { Néolibéralisme } \\
\text { économique }\end{array}$ & $\mathrm{X}$ & $\mathrm{X}$ & $\mathrm{X}$ & $\mathrm{X}$ & $\mathrm{X}$ \\
\hline $\begin{array}{l}\text { Démocratie et } \\
\text { droits }\end{array}$ & $\mathrm{X}$ & $\mathrm{X}$ & $\mathrm{X}$ & $\mathrm{X}$ & $\mathrm{X}$ \\
\hline $\begin{array}{l}\text { Culture et } \\
\text { communication }\end{array}$ & $\mathrm{X}$ & $\mathrm{X}$ & $\mathrm{X}$ & $\mathrm{X}$ & $\mathrm{X}$ \\
\hline $\begin{array}{l}\text { Environnement } \\
\text { et développement }\end{array}$ & $\mathrm{X}$ & $\mathrm{X}$ & $\mathrm{X}$ & $\mathrm{X}$ & $\mathrm{X}$ \\
\hline $\begin{array}{l}\text { Groupes } \\
\text { particuliers }\end{array}$ & $\mathrm{X}$ & $\mathrm{X}$ & & & $\mathrm{X}$ \\
\hline Religion & $\mathrm{X}$ & & & $\mathrm{X}$ & \\
\hline Migrations & & & & & $\mathrm{X}$ \\
\hline
\end{tabular}

Ainsi, la catégorie culture et communication renvoie plus généralement à l'univers de la culture, de l'identité, que ce soit dans le domaine des arts, des pratiques, des médias ou des savoirs. Les questions de la diversité culturelle et de sa préservation ainsi que de la capacité d'expression occupent une place centrale dans cette thématique, laquelle est traitée dans tous les forums et trai- 
tée comme axe primordial, exception faite du Forum social européen qui ne l'aborde que comme un sous-thème de son axe « Justice sociale et solidarité ». Ainsi, sans pour autant être absente du FSE de Londres, cette thématique de la communication n'est pas réellement centrale.

On a le phénomène similaire avec la catégorie environnement et développement, traitée comme un sous-thème de la thématique économique (comme conséquence de l'activité économique) et de l'impérialisme (contrôle stratégique de la biodiversité) lors du Forum social des Amériques, alors que ces questions figurent comme des axes thématiques distincts dans les autres forums étudiés. Encore une fois, cela ne signifie pas que ce thème n'était pas traité, mais qu'il occupait une position de second ordre dans le FSA de Quito.

\section{Les thèmes régionaux}

C'est dans l'identification de thématiques particulières à certains forums que l'analyse se révèle plus intéressante. En effet, certaines catégories ne se retrouvent que dans certains forums, ce qui laisse supposer une forme de régionalisme dans ces thématiques. Nous avons pu identifier trois thématiques spécifiques.

Tout d'abord, la catégorie des groupes particuliers. En effet, l'identification de ces groupes spécifiques faisant l'objet de discriminations a été très clairement présente lors du Forum social mondial de Mumbai en 2004. En effet, les questions des castes, du patriarcat et des minorités de toutes sortes ont figuré en première ligne. D'ailleurs, les questions de genre et de diversité y ont été considérées comme des axes transversaux. De même pour le Forum social des Amériques où les peuples indigènes et afro-américains ont fait l'objet d'un axe thématique distinct. Enfin, lors du Forum social de la Méditerranée, la question des femmes a fait, elle aussi, l'objet d'un axe en soi.

Ensuite, la thématique religieuse est apparue clairement dans les deux forums sociaux mondiaux (2004 et 2005), alors qu'elle ne semble centrale dans aucun des trois autres forums étudiés. Dans le cas du forum indien, la religion est liée à la problématique identitaire et culturelle, alors que dans le cas du FSM de Porto Alegre, elle constitue un axe à part, se déclinant, selon la formule adoptée en "éthique, cosmovision et spiritualité ». L'importance du facteur religieux dans la culture indienne, de même que dans la culture politique brésilienne (pensons à la théologie de la libération ${ }^{42}$ ) doit ici constituer un facteur de distinction.

Enfin, on a la catégorie des migrations qui, elle, est très clairement attachée au Forum social méditerranéen. Mais cela ne doit pas nous surprendre tant

42. La théologie de la libération est le nom donné à un mouvement social et religieux issu de l'Eglise catholique apparu en Amérique latine, notamment au Brésil, au début des années 1960 
cette question est centrale dans la région, la Méditerranée constituant une ligne de fracture économique et sociale entre le Nord et le Sud.

Cette première analyse qualitative sommaire des thématiques abordées lors des cinq forums sociaux retenus ne nous permet cependant pas d'aboutir à l'affirmation tranchée d'une régionalisation claire des forums sociaux. Elle nous permet, tout au plus, d'énoncer un certain nombre de nuances dans les priorités d'action et les problèmes rencontrés. Elle révèle cependant la diversité des contextes socio-historiques et des réalités vécues. La question des castes, des peuples autochtones, le souci de l'environnement, les migrations, la place des femmes voire les conceptions du travail et du développement peuvent varier d'un continent à l'autre, voire ne pas constituer du tout de véritable objet de lutte ou de mobilisation. En somme, si tous peuvent s'entendre dans la lutte contre le néolibéralisme et l'impérialisme, et pour la défense des droits, de la dignité et de la diversité, les formes concrètes des problèmes sociaux qui se posent et des solutions qu'il convient d'y apporter peuvent être profondément différentes suivant les régions du monde.

\section{Analyse des déclarations}

Si l'analyse des axes thématiques nous a permis de révéler des manières différentes, suivant les régions du monde, de vivre les conséquences de la mondialisation, l'étude des déclarations adoptées par les assemblées des mouvements sociaux lors de ces forums nous permet cette fois-ci de rendre compte des formes d'action proposées afin de rendre possible un autre monde. Une précision d'importance doit être introduite ici. Selon l'article 6 de la Charte des principes du FSM :

« les rencontres du Forum social mondial n’ont pas un caractère délibératif en tant que Forum social mondial. Personne ne sera donc autorisé à exprimer au nom du Forum, dans quelque édition que ce soit, des prises de position prétendant être celles de tous les participants. [...] Il ne constitue donc pas d'instance de pouvoir que peuvent se disputer ceux qui participent à ces rencontres, ni ne prétend constituer l'unique alternative d'articulation et d'action des instances qui en font partie ».

Compte tenu de cette posture ontologique qui conduit à concevoir les forums sociaux comme des espaces de rassemblement de la mouvance altermondialiste et

lorsque des catholiques progressistes s'éloignent d'un catholicisme conservateur au profit d'une voie dans laquelle l'action politique apparaît comme une exigence de l'engagement religieux dans la lutte contre la pauvreté. Théorisé au début des années 1970, ce courant théologique parfois teinté de marxisme, prône la libération des peuples et entend ainsi renouer avec la tradition chrétienne de solidarité. Löwy M., « La théologie de la libération : Leonardo Boff et Frei Betto ", Réseau d'information et de solidarité avec l'Amérique latine - RISAL, 14 mars 2007, http://risal.collectifs.net/ 
non comme des acteurs politiques en soi ${ }^{43}$, les forums ne débouchent pas sur une déclaration finale du forum. En revanche, différentes coalitions d'acteurs peuvent adopter une déclaration commune, comme c'est le cas pour les assemblées des mouvements sociaux, qui n'engagent cependant que les membres de ces assemblées et non pas l'ensemble des participants du forum social. Comment pourraitil d'ailleurs en être autrement sans bafouer les principes de diversité et de participation ? Cela dit, les assemblées des mouvements sociaux réunissent généralement de larges coalitions d'organisations et de mouvements (syndicats, ONG, mouvements de femmes, groupes citoyens, associations paysannes, etc.) dotées d'une forte capacité d'action. Certes, les registres d'action prônés par ces mouvements traditionnels demeurent assez classiques et ne rendent pas compte de la diversité et de la créativité dont font preuve toutes sortes d'autres minorités actives ${ }^{44}$ cherchant à renouveler les pratiques contestataires en investissant les forums sociaux. Il n'en demeure pas moins que, sans refléter la richesse des multiples propositions d'action qui émanent des forums sociaux, les déclarations de ces assemblées constituent une première porte d'entrée pour tester notre hypothèse. Ainsi, nous analyserons successivement chacune des déclarations des mouvements sociaux présentées lors des forums sélectionnés 45 en distinguant d'une part les échelles d'action proposées et, d'autre part, les registres d'action ${ }^{46}$.

\section{Les échelles d'action}

L'Appel de l'Assemblée des monvements socianx lancé lors du Forum social mondial de Mumbai en 2004 se situe clairement dans un contexte global de domination, caractérisé par l'articulation évidente entre le néolibéralisme et l’impérialisme depuis le début de la guerre en Irak (mars 2003). Que ce soit par

43. Ceci constitue un débat de fond au sein de la mouvance altermondialiste. Voir Teivainen T., “The World Social Forum : arena or actor?", in Sen J., Anand A., Escobar A., Waterman P., World Social Forum: Challenging Empires, New Delhi, The Viveka Foundation, 2004, pp. 122-129.

44. Sommier I., Le Renouveau des mouvements contestataires à l'beure de la mondialisation, Paris, Flammarion, 2003.

45. Il s'agit pour le Forum social mondial de Mumbai de l'Appel de l'Assemblée des mouvements sociaux (Mumbai, 27 janvier 2004) ; pour le Forum social des Amériques de la Declaración del foro: El nuevo liberalismo y sus facetas (Quito, 25 juillet 2004) ; pour le Forum social européen de l'Appel de l'Assemblée des mouvements sociaux (Londres, 17 octobre 2004); pour le Forum social mondial de Porto Alegre de l'Appel des mouvements sociaux à la mobilisation contre la guerre, le néolibéralisme, l'exploitation et l'exclusion pour un autre monde possible (Porto Alegre, 31 janvier 2005) ; et enfin pour le Forum social méditerranéen de Barcelone, de l'Appel de l'assemblée des mouvements sociaux de la Méditerranée (Barcelone, 19 juin 2005). Tous ces textes sont disponibles sur Internet.

46. Nous nous référons ici à l'analyse de cadres (frame analysis), inspirée des travaux précurseurs d'Erwin Goffman, qui fut développée dans le champ de la sociologie de l'action collective et des mouvements sociaux autour de Charles Tilly, Sidney Tarrow, David Snow et Robert Benford. Elle permet le développement d'une approche cognitive des phénomènes sociaux qui s'inspire de la sociologie compréhensive wébérienne et du courant de l'interactionnisme symbolique. Voir Cefai D., Tromp D. (dirs.), Les Formes de l'action collective. Mobilisations dans les arènes publiques, Paris, Editions de l'EHESS, 2001 ; Chazel F. (dir.), Action collective et mouvements sociaux, Paris, PUF, 1993. 
les accords de libre-échange, les négociations commerciales à l'OMC, le travail des institutions financières internationales (FMI, Banque mondiale) et, désormais, la guerre, le militarisme et l'obsession sécuritaire (au nom de la lutte contre le terrorisme), le système néolibéral tente de s'imposer partout, entraînant dans son sillage son cortège de destructions et d'injustices. Face à ce régime global de domination, l'Appel vise à tisser un lien entre toutes les luttes sociales qui se manifestent partout sur la planète. Les mouvements sociaux réunis lors de cette assemblée entendent donc se situer dans la filiation de luttes locales et spécifiques (au Chiapas mexicain, contre l'OMC à Seattle et Cancún, contre le G8 à Gênes, les marches contre la guerre en Irak du 15 février 2003, etc.) et souhaitent les soutenir et les renforcer en leur donnant une portée internationale (par l'organisation de journées internationales de mobilisation à dates fixes). C'est en somme une action glocale qui est promue par cette déclaration et qui s'articule selon la séquence suivante : une conscience globale du problème (le couple néolibéralisme/impérialisme); le constat d'une multiplicité de manifestations locales et spécifiques de formes de résistances et d'actions ; la nécessité de fédérer ces luttes ; l'organisation de mobilisations internationales pour soutenir et propager ces luttes. Dans cette perspective, le FSM apparaît comme une caisse de résonance de toutes les luttes locales et spécifiques parcourant la planète.

La Déclaration du forum sur le nouveau libéralisme et ses facettes, ressortie du Forum social des Amériques de Quito, se situe quant à elle clairement dans le contexte continental américain, et plus spécifiquement dans le contexte latino-américain. En effet, un clivage marqué apparaît entre « l'impérialisme nord-américain » et «nos peuples », entendus d'Amérique latine. Dans cette configuration, les signataires de cette déclaration souhaitent la constitution d'un grand mouvement bolivarien latino-américain qui permettrait d'unifier l'Amérique latine, afin de la constituer en une puissance indépendante et souveraine, conformément au rêve de Simon Bolívar ${ }^{47}$. Ils en appellent ainsi à la constitution d'un bloc unifié de pouvoir sous-régional capable de faire face aux visées hégémoniques de «l'empire yankee». Dans cette déclaration, la dimension antagoniste est clairement exprimée car l'ennemi est identifié, et sa dénonciation repose sur une longue série de faits historiques. Par ailleurs, l'échelle d'action paraît d'autant plus évidente qu'elle est déjà promue par un projet politique à tendance sous-régionale mis en avant par le Venezuela : l'Alternative bolivarienne pour les Amériques (ALBA) 48.

47. Canet R., «Intégration ou désintégration continentale ? Les Amériques entre hégémonie et diversité », in Lesemann F., Côté J.-F., La Construction des Amériques aujourd'bui : regards croisés transnationaux et transdisciplinaires, Québec, Presses universitaires du Québec, à paraitre.

48. L’ALBA est une initiative lancée par le président vénézuélien Hugo Chávez et inspirée de la lutte du Libertador Simon Bolivar. L'ALBA se présente comme une alternative d'intégration au projet de Zone de libre-échange des Amériques (ZLEA) lancé par les Etats-Unis. Elle est officiellement née à La Havane, en avril 2005. Un an plus tard, la Bolivie d'Evo Morales a rejoint cette alliance en proposant notamment un Traité commercial des peuples (en opposi- 
L'Appel des monvements socianx du Forum social européen de Londres se situe lui aussi dans une perspective régionale (l'Europe), même s'il fait le lien avec des problématiques plus globales (notamment la guerre et les changements climatiques). Il énonce toute une série de revendications (contre la violence envers les femmes, le racisme, le chômage et la précarité, pour les droits des migrants, la diversité culturelle, l'égalité, la protection sociale, etc.) afin de permettre la construction d'une "autre Europe », opposée au «modèle néolibéral européen » qui semble promu par le Traité constitutionnel européen, alors dans sa phase de ratification ${ }^{49}$. Notons qu'au moment du FSE de Londres, la question de la Constitution européenne était un sujet brûlant d'actualité. En effet, le Traité ayant été adopté par les chefs d'Etat et de gouvernement des pays membres de l'Union lors du Conseil européen de Bruxelles des 17 et 18 juin 2004, il est formellement signé à Rome le 29 octobre, soit un peu plus de dix jours après la tenue du FSE. Cependant, puisque son entrée en vigueur était conditionnée par la ratification de la totalité des membres de l'Union, un large débat public a été lancé, plus spécifiquement dans les pays comme la France, les Pays-Bas, l'Espagne ou le Luxembourg, qui ont choisi de consulter leurs populations sur cette question et donc d'entrer dans une phase de campagne électorale.

\section{L'Appel des mouvements sociaux à la mobilisation contre la guerre, le néolibé-} ralisme, l'exploitation et l'exclusion pour un autre monde possible, du Forum social mondial de Porto Alegre de 2005, tout comme celui du FSM 2004, adopte une approche glocale du changement social en réaffirmant l'articulation nécessaire entre la mouvance globale et les luttes populaires locales. Ce concept permet d'insister sur le fait que les multiples mouvements, organisations et coalitions de toutes natures vont déployer des stratégies d'action sur un continuum s'étalant du micro au macro, des communautés de base, voire de l'individu jusqu'à la communauté internationale, du quartier à l'ONU. Cette approche constitue d'ailleurs la particularité de ces nouvelles formes de mobilisation sociale puisque «la nouveauté de notre époque est l'explosion et l'extension des mouvements sociaux sur tous les continents et leur capacité à construire dans la diversité, des convergences nouvelles et des actions communes au niveau global 50 ». Puisque la problématique est globale, mais que les acteurs de transformation sociale sont ancrés locale-

tion aux traités de libre-échange) entre ses membres. Le Nicaragua, présidé par Daniel Ortega, a rejoint l'alliance en janvier 2007. L'ALBA entend se baser sur les principes de solidarité, de coopération et de complémentarité. L'initiative s'oppose notamment à la suppression des droits de douane et propose la création de fonds compensatoires et l'utilisation de commandes publiques privilégiant les coopératives et les petites ou les moyennes industries. L'ALBA prétend également s'attaquer aux principales faiblesses du continent : l'insuffisance énergétique (en créant PETROSUR) et le monopole de l'information (avec TELESUR). Source : Centre tricontinental de Louvain (Belgique) http://www.cetri.be/

49. Au moment du FSE de Londres, les Pays-Bas et la France n'avaient pas encore exprimé, par voie référendaire, leur opposition au projet de Constitution européenne, minant ainsi le processus de ratification du Traité.

50. Appel des mouvements sociaux à la mobilisation contre la guerre, le néolibéralisme, l'exploitation et l'exclusion pour un autre monde possible, Porto Alegre, 31 janvier 2005. 
ment, l'action glocale consiste en une relation itérative du global au local permettant de surmonter le défi de la convergence dans la diversité.

\section{L'Appel de l'assemblée des mouvements sociaux de la Méditerranée (FSMed} 2005) se réfère à la région méditerranéenne qui rassemblerait l'ensemble des peuples se situant sur ses rives. Cependant, au-delà de la référence à cette entité géographique comme univers partagé, l'Appel ne définit pas d'échelle d'action régionale précise. En réalité, il énonce une série de prises de position sur des questions fondamentales qui affectent plus particulièrement certaines parties de cet espace méditerranéen, notamment la guerre en Irak, les revendications du peuple palestinien, la dette des pays du Sud, la directive Bolkestein ${ }^{51}$ et la question du Traité constitutionnel en Europe. Ainsi, au-delà du slogan rassembleur invoquant «une mer de paix et de droits » pour tous et de grands énoncés de principes (démocratie, laïcité, lutte contre la discrimination et le patriarcat...), ce sont plutôt de multiples échelles d'action qui semblent se dessiner dans cet Appel, ce qui rend compte à la fois de la diversité des problèmes rencontrés, mais aussi de la croyance que ce sont des actions locales qui pourront faire évoluer les choses. Les seuls problèmes qui semblent transcender les frontières et fournir les bases d'une véritable action à l'échelle de la Méditerranée sont ceux des migrations et des accords de libre-échange, notamment dans le cadre du Partenariat euroméditerranéen ${ }^{52}$, qui a justement été signé à Barcelone, où s'est tenu le FSMed, dix ans plus tôt. Finalement, nous pouvons supposer que le flou entretenu autour de l'échelle d'action semble aussi lié à la représentation problématique de cet espace maritime, tantôt « part du milieu » qui fut historiquement un carrefour de civilisations ${ }^{53}$, tantôt « limes » délimitant une zone de confrontation et de fracture entre «l'Empire et les nouveaux barbares ${ }^{54}$ ».

\section{Les registres d'action}

Dans leur logique d'action glocale, reposant sur une publicisation des luttes locales au niveau international, les assemblées des mouvements sociaux qui se sont tenues lors des Forums sociaux mondiaux de Mumbai (2004) et de Porto Alegre (2005) débouchent principalement sur des appels à des mobilisa-

51. Du nom du Commissaire européen qui en fut l'artisan, cette directive européenne visait la libéralisation du secteur des services dans le marché intérieur de l'Union.

52. Le partenariat euroméditerranéen, dit " processus de Barcelone », regroupe les Etats membres de l'Union européenne et dix Etats du Sud et de l'Est de la Méditerranée (Maroc, Algérie, Tunisie, Egypte, Israël, Autorité palestinienne, Jordanie, Liban, Syrie et Turquie). Il comporte trois volets : 1 - économique et financier; 2 - politique 3 - culturel, social et humain. C’est le premier volet qui est de loin le plus développé et, dans la perspective de la création d'une zone de libre-échange en 2010, une dizaine d'accords d'association ont été conclus afin de développer les échanges entre l’UE et les pays méditerranéens.

53. Braudel F., La Méditerranée et le monde méditerranéen à l'époque de Philippe II, Paris, Armand Colin, 1949.

54. Pour une approche critique de cette théorie du limes, voir Rufin J.-C., L'Empire et les nouveaux barbares, Paris, Lattès, 1992. 
tions internationales ponctuelles sur des enjeux spécifiques. Huit rendez-vous militants sont ainsi annoncés à Mumbai : la journée internationale pour les droits des femmes (8 mars); la journée internationale de lutte paysanne (17 avril) ; la journée internationale contre la guerre et l'occupation de l'Irak (20 mars) ; la journée de la Terre en Palestine (30 mars) ; la journée de mobilisation pour l'inclusion sociale ; mais aussi les mobilisations contre le G8, le FMI et la Banque mondiale ; contre l'OMC ; contre la privatisation des biens communs et des ressources naturelles.

L'Appel de Porto Alegre détaille lui aussi tout un calendrier des luttes et mobilisations, et invite à la solidarité avec une série de campagnes (pour l'annulation de la dette des pays du Sud; contre les bases militaires américaines dans le monde; contre le patriarcat, l'exclusion et la domination avec la marche mondiale des femmes; pour la défense de l'eau comme bien commun; contre les firmes multinationales, pour les droits des enfants et contre l'exploitation sexuelle, etc.), ainsi qu'avec une journée d'action globale contre la guerre en Irak (les 19 mars de chaque année depuis 2003) ; contre les accords de libre-échange (durant les journées d'action globale des 10 au 17 avril 2005, puis en marge du Sommet des Amériques à Mar del Plata, Argentine, les 4 et 5 novembre 2005 et, finalement, lors de la conférence ministérielle de l'OMC à Hong Kong, Chine, du 13 au 18 décembre 2005); contre le G8 (du 2 au 8 juillet 2005 à Gleneagles, en Ecosse); contre le 4e Sommet Union européenne - Amérique latine et Caraibes (du 11 au 13 mai 2006 à Vienne, en Autriche); pour la journée mondiale des luttes paysannes (célébré tous les 17 avril depuis 1996) ainsi que pour le jour anniversaire de la mort de Lee Kyung-Hae, le leader paysan coréen qui s'est suicidé en signe de protestation le 10 septembre 2003 lors de la conférence de l'OMC à Cancún (Mexique); pour un nouvel ordre mondial démocratique ; contre la pauvreté et la guerre ; pour la journée internationale des étudiants... Tous ces appels à la solidarité internationale envers des luttes locales ou spécifiques débouchent finalement sur des formes classiques de mobilisation prenant généralement l'aspect soit de célébration de journées thématiques consacrées, soit de manifestations et de contre-sommets lors d'événements ponctuels. Il s'agit, en investissant la rue, d'exprimer massivement ses désaccords avec les politiques mises en œuvre par les élites économico-politiques et de publiciser largement des situations d'injustice.

Le contexte géopolitique continental dans lequel s'inscrit la Déclaration $d u$ Forum sur le nouveau libéralisme et ses facettes (FSA 2004) est très clairement décrit comme profondément antagoniste, voire guerrier (il est fait référence à la Quatrième Guerre mondiale annoncée par le sous-commandant Marcos dès 1997 55). L'Amérique latine est fortement menacée par l'impérialisme nordaméricain et doit prendre des mesures drastiques pour organiser sa défense. Les

55. Sous-commandant Marcos, «La Quatrième Guerre mondiale a commencé », Le Monde diplomatique, août 1997, p. 1 et pp. 4-5. 
Etats-Unis, puissance impérialiste, sont accusés de vouloir destituer les pouvoirs en place, voire envahir militairement la République bolivarienne du Venezuela, l'Argentine et le Brésil. Sont aussi dénoncés les gouvernements jugés serviles d'Alvaro Uribe en Colombie, et de Lucio Gutiérrez en Equateur. Cette pression militaire de la part du Nord du continent se double d'une offensive sur le plan économique, avec les accords de libre-échange (le projet de Zone de libreéchange des Amériques, ZLEA, puis les accords bilatéraux signés par les EtatsUnis) qui conduisent au pillage des ressources naturelles et culturelles (notamment par le dépôt de brevets visant l'appropriation des savoirs ancestraux). Afin de lutter contre cet impérialisme, les signataires de cette déclaration s'en remettent aux gouvernements dits «progressistes " (Castro, Chavez, Lula, Kirchner) afin de soutenir un projet socialiste d'unification de l'Amérique latine, de mettre sur pied une armée bolivarienne unifiée capable de se défendre à l'échelle sous-régionale, de légaliser la drogue pour en finir avec le narcotrafic. Ils en appellent aussi à la désobéissance civile contre les législations préjudiciables aux conditions de vie des peuples latino-américains, au boycott des produits des grandes multinationales. Finalement, ils souhaitent que se constitue une véritable entité politique latino-américaine intégrée avec une constitution socialiste, une monnaie, un Parlement, un drapeau et un hymne propres.

L'Appel des mouvements sociaux du Forum social européen de Londres adopte un registre revendicatif assez similaire à ce que nous avons pu observer dans les cas des deux FSM. Il dénonce une série d'injustices et invite à des mobilisations internationales de toutes natures (contre le «mur de l'apartheid » en Palestine, du 9 au 16 novembre 2005 ; pour les droits de l'Homme lors des journées d'action européennes, les 10 et 11 décembre 2005 ; contre le sommet de l'OTAN, du 9 au 12 février 2005 ; contre le G8, du $1^{\text {er }}$ au 8 juillet 2005 ; contre les violences faites aux femmes, tous les 25 novembre depuis $1999 . .$.$) Des mobilisations plus spécifiques aux réalités européennes$ sont aussi annoncées, notamment dans le contexte de ratification du Traité constitutionnel européen (contre le Traité constitutionnel, 30 octobre 2005 ; contre la directive Bolkestein, 11 novembre 2005 ; contre le racisme et pour le droit des migrants, 2 avril 2005, etc.). Là encore, la stratégie semble s'articuler autour de la mise en scène d'un rapport de force entre la rue et les gouvernements (la Commission européenne est directement interpellée), entre le peuple et ses élites, par l'organisation de manifestations de masse autour de revendications spécifiques ou en marge de rencontres officielles.

Finalement, le caractère plus ou moins tangible ou, tout du moins, fortement hétérogène de la région méditerranéenne couverte par le FSMed et la diversité des échelles d'action locales qui découlent de cette vision, n'est pas sans conséquence sur les modalités d'action énoncée dans l'Appel de l'assemblée des mouvements sociaux de la Méditerranée. En effet, dans cette région soumise à "l'agression néolibérale » et aux "projets impérialistes », les mouvements sociaux des rives de la mare nostrum doivent entrer en résistance afin 
de défendre les droits des «travailleurs, des citoyens et des peuples». Elément notable et significatif de cette déclaration, elle ne se limite pas aux mouvements sociaux, mais inclus également les individus, hommes, femmes et jeunes qui ont pris part au forum. C'est donc principalement une action par la base qui est mise en avant puisque, finalement, la seule forme concrète de structuration proposée consiste dans l'organisation de réseaux de solidarité et la coordination entre les mouvements sociaux. Cette stratégie de résistance par la base, qui consiste principalement à manifester son opposition et à revendiquer des droits, se retrouve d'ailleurs dans l'Agenda des mobilisations annexé à l'Appel de l'assemblée et qui énonce, une fois encore, toute une série de campagnes de protestation (contre la guerre, contre la zone de libre-échange, contre le G8 et la dette, contre l'OMC, etc.).

\section{Une nouvelle culture politique pour refonder la démocratie}

La mouvance altermondialiste, telle qu'elle se laisse saisir dans ses multiples lieux de manifestation, apparaît telle un kaléidoscope de revendications et de propositions qui entendent à la fois rendre compte de la diversité des manières de vivre et réagir au phénomène de la mondialisation néolibérale. En ce sens, elle prolonge la dynamique particulariste qui caractérise les mouvements sociaux dans les sociétés post-industrielles ${ }^{56}$. Dans cette perspective, les dynamiques d'expansion (délocalisation, diversification) et d'approfondissement (multiplication, déclinaison) des forums sociaux ont stimulé cette diversité des expressions de la contestation mondiale en multipliant les espaces publics critiques ${ }^{57}$. Cela débouche sur une série de perspectives.

Tout d'abord, sur le plan de la représentation, cette diversité des thématiques, des échelles et des registres d'action ne fait que suggérer qu'il n'y a pas une seule solution possible à la mondialisation néolibérale car celle-ci ne se manifeste pas de la même manière dans toutes les parties du globe et ses conséquences sont très variables selon les diverses populations qui en sont affectées. L'erreur serait de penser le forum comme un acteur qui aurait un discours global cohérent et contre-hégémonique ${ }^{58}$. Il s'agit plutôt d'un espace donnant lieu, à chaque endroit où il se manifeste, à l'expression de formes particulières de lutte et de solidarité 59 .

56. Farro A.L., Les Mouvements sociaux : diversité, action collective et globalisation, Montréal, Presses de l'université de Montréal, 2000.

57. Sans qu'il traite directement de la mouvance altermondialiste, nous renvoyons ici malgré tout à l'ouvrage d'O. Negt, L'Espace public oppositionnel, Paris, Payot, 2007, qui développe ce concept fécond où peuvent s'exprimer toutes les «subjectivités rebelles ». Le parallèle avec la multitude de la mouvance altermondialiste investissant les forums sociaux serait à explorer.

58. La tentation est pourtant grande au sein de la mouvance, voir Sen J., Bond P., A Political Programme for the World Social Forum?, New Delhi/Durban, CACIM-CCS, janvier 2007.

59. Fisher W., Ponniah T., Another World is Possible. Popular Alternatives to Globalization at the World Social Forum, New York, Zed Books, 2003. 
Ensuite, sur le plan de l'action, la diversité des échelles et des stratégies proposées par les multiples acteurs sociaux qui se rassemblent dans les forums, permet de relativiser la localisation exclusive des initiatives alternatives et, ainsi, de laisser ouvert le champ des possibles. Cette remarque n'est pas sans lien avec la précédente, elle en découle directement : c'est parce que la mondialisation néolibérale et l'altermondialisme sont tous deux perçus comme complexes, multiformes et multiscalaires, qu'il apparait légitime, comme nous le suggère Ulrich Beck ${ }^{60}$, de nous émanciper du "nationalisme méthodologique " pour penser ces phénomènes contemporains. Cela ne doit cependant pas nous conduire à opter de manière exclusive pour les paradigmes soit globalistes, soit localistes. Comme semble le suggérer l'analyse discursive des forums sociaux, l'échelle d'action est plutôt glocale. Dès lors, il ne s'agit pas simplement de penser globalement et d'agir localement ${ }^{61}$, mais bien de penser et d'agir à tous les niveaux.

Il convient toutefois de noter que cet éloge de la diversité relève également d'une stratégie discursive de lutte contre ce que les tenants de la mouvance altermondialiste nomment « la pensée unique » légitimant un mode d'organisation politique des sociétés versant dans l'élitisme et la technocratie. Dans cette perspective, les forums sociaux se présentent comme les catalyseurs d'une nouvelle culture politique visant à refonder la démocratie sur de nouvelles bases, plus participatives.

Cela nous amène à revenir sur les différents registres d'action que nous avons dégagés de l'analyse des déclarations des assemblées des mouvements sociaux. Rappelons préalablement que ces registres ne constituent que des échantillons très limités des propositions d'actions qui émanent des forums et qu'ils ne sont en rien représentatifs de la mouvance dans son ensemble. Ils permettent cependant d'illustrer la diversité des actions menées selon une variable que nous souhaiterions aborder, celle de la culture politique de l'aire géopolitique considérée.

En effet, les assemblées des mouvements sociaux des deux FSM et du FSE semblent privilégier des registres d'action assez classiques, sous la forme de larges mobilisations collectives, de manifestations, visant à interpeler les classes dirigeantes, à faire pression sur les pouvoirs institutionnalisés. Nous sommes ici dans le registre d'action traditionnel des mouvements sociaux occidentaux, principalement le mouvement ouvrier ${ }^{62}$. Pour le cas du FSA, le registre est plus radical et semble très marqué par la réalité sociopolitique latino-américaine actuellement très polarisée. Il se situe très clairement dans la perspective de la révolution bolivarienne et se fonde sur une stratégie de conquête du pouvoir étatique afin de

60. Beck U., op. cit.

61. ATTAC, Agir local, penser global, Paris, Mille et une nuits, 2001.

62 . Nous renvoyons ici à l'œuvre d'Alain Touraine et ses successeurs au CADIS de l'Ecole des hautes études en sciences sociales (EHESS) de Paris. 
bâtir un bloc continental qui puisse contrer les velléités hégémoniques des EtatsUnis. Rappelons que l'Amérique latine est le berceau des forums sociaux et que la culture politique y est extrêmement militante et engagée. Finalement, pour le cas du FSMed, nous sommes au stade premier de la construction d'une coalition de mouvements sociaux par la base. Avant même d'être à même de conquérir le pouvoir comme en Amérique latine, ou de se positionner tel un contre-pouvoir comme en Europe, encore faut-il s'être constitué en un réseau d'acteurs sociaux mobilisés et mobilisables. Encore faut-il également disposer d'une société civile consciente de ses droits et capable de se mobiliser pour les revendiquer. Encore faut-il, enfin, accéder à un espace pour pratiquer cette nouvelle culture politique participative. Dans cette perspective, la multiplication des initiatives visant à organiser des forums sociaux, prenons par exemple le cas du Forum social maghrébin ${ }^{63}$, apparaît comme un moyen pour la mouvance altermondialiste d'approfondir un processus de démocratisation en ouvrant des espaces publics critiques de participation citoyenne. 